University of Texas at El Paso

ScholarWorks@UTEP

\title{
Plans Are Worthless but Planning Is Everything: A Theoretical Explanation of Eisenhower's Observation
}

\author{
Angel F. Garcia Contreras \\ The University of Texas at El Paso, afgarciacontreras@miners.utep.edu \\ Martine Ceberio \\ The University of Texas at El Paso, mceberio@utep.edu \\ Vladik Kreinovich \\ The University of Texas at El Paso, vladik@utep.edu
}

Follow this and additional works at: https://scholarworks.utep.edu/cs_techrep

Part of the Computer Sciences Commons

Comments:

Technical Report: UTEP-CS-17-16

To appear in Proceedings of the 10th International Workshop on Constraint Programming and Decision Making CoProd'2017, El Paso, Texas, November 3, 2017.

\section{Recommended Citation}

Garcia Contreras, Angel F.; Ceberio, Martine; and Kreinovich, Vladik, "Plans Are Worthless but Planning Is Everything: A Theoretical Explanation of Eisenhower's Observation" (2017). Departmental Technical Reports (CS). 1102.

https://scholarworks.utep.edu/cs_techrep/1102

This Article is brought to you for free and open access by the Computer Science at ScholarWorks@UTEP. It has been accepted for inclusion in Departmental Technical Reports (CS) by an authorized administrator of ScholarWorks@UTEP. For more information, please contact Iweber@utep.edu. 


\title{
Plans Are Worthless but Planning Is Everything: A Theoretical Explanation of Eisenhower's Observation
}

\author{
Angel F. Garcia Contreras, Martine Ceberio, and Vladik Kreinovich \\ Department of Computer Science, University of Texas at El Paso \\ El Paso, TX 79968, USA \\ afgarciacontreras@miners .utep.edu, \\ mceberio@utep.edu, vladik@utep.edu
}

\begin{abstract}
The 1953-1961 US President Dwight D. Eisenhower emphasized that his experience as the Supreme Commander of the Allied Expeditionary Forces in Europe during the Second World War taught him that "plans are worthless, but planning is everything". This sound contradictory: if plans are worthless, why bother with planning at all? In this paper, we show that Eisenhower's observation has a meaning: while directly following the original plan in constantly changing circumstances is often not a good idea, the existence of a pre-computed original plan enables us to produce an almost-optimal strategy - a strategy that would have been computationally difficult to produce on a short notice without the pre-existing plan.
\end{abstract}

\section{Introduction: Eisenhower's Seemingly Paradoxical Observation}

Eisenhower's observation. Dwight D. Eisenhower, the Supreme Commander of the Allied Expeditionary Forces in Europe during the Second World War and later the US President, emphasized that his war experience taught him that "plans are worthless, but planning is everything"; see, e.g., [1].

At first glance, this observation seems paradoxical. At first glance, the Eisenhower's observation sounds paradoxical: if plans are worthless, why bother with planning at all?

What we do in this paper. In this paper, we show that this Eisenhower's observation has a meaning. Namely, it means that:

- while following the original plan in constantly changing circumstances is often not a good idea,

- the existence of a pre-computed original plan enables us to produce an almost-optimal strategy (a strategy that would have been computationally difficult to produce on a short notice without the pre-existing plan). 


\section{Analysis of the Problem}

Rational decision making: a brief reminder. According to decision making theory, decisions by a rational decision maker can be described as maximize the value a certain function known as utility; see, e.g., [3,4]. In financial situations, when a company needs to make a decision, the overall profit can be used as the utility value; in more complex situations, the utility function combines different aspects of gain and loss related to different decisions.

Let us describe this in precise terms. Let $x$ denote a possible action, $a$ describes the situation, and let $u(x, a)$ denote the utility that results from performing action $x$ in situation $a$.

To describe a possible action, we usually need to describe the values of several different quantities. For example, a decision about a plant involves selecting amount of gadgets of different type manufactured at this plant - and maybe also the parameters characterizing these gadgets. Let us denote the parameters describing an action by $x_{1}, \ldots, x_{n}$. In these terms, an action can be characterized by the tuple $x=\left(x_{1}, \ldots, x_{n}\right)$.

Similarly, in general, we need several different quantities to describe a situation, so we will describe a situation by a tuple $a=\left(a_{1}, \ldots, a_{m}\right)$.

In these terms, what is planning. Let $\widetilde{a}$ describe the original situation. Based on this situation, we come up with an action $\widetilde{x}$ that maximizes the corresponding utility: $u(\widetilde{x}, \widetilde{a})=\max _{x} u(x, \widetilde{a})$. Computing this optimal action $\widetilde{x}$ is what we usually call planning.

Situations change. At the moment when we need to start acting, the situation may have changed in comparison with the original situation $\widetilde{a}$, to a somewhat different situation $a$. Let us denote the corresponding change by $\Delta a \stackrel{\text { def }}{=} a-\widetilde{a}$. In terms of this difference, the new situation takes the form $a=\widetilde{a}+\Delta$.

A not-always-very-good option: applying the original plan to the new situation. One possibility is to simply ignore the change, and apply the original plan $\widetilde{x}$ - which was optimal for the original situation $\widetilde{a}-$ to the new situation $a=\widetilde{a}+\Delta a$.

This plan is, in general, not optimal for the new situation. Thus, in comparison to the actually optimal plan $x^{\text {opt }}$ for which

$$
u\left(x^{\mathrm{opt}}, \widetilde{a}+\Delta a\right)=\max _{x} u(x, \widetilde{a}+\Delta a),
$$

we lose the amount $L_{0} \stackrel{\text { def }}{=} u\left(x^{\text {opt }}, \widetilde{a}+\Delta a\right)-u(\widetilde{x}, \widetilde{a}+\Delta a)$.

A better option: trying to modify the original plan. Why cannot we just find the optimal solution for the new situation? Because optimization is, in general, an NP-hard problem (see, e.g., $[2,5]$ ), meaning that it is not possible to find the exact optimum in reasonable time.

What we can do is try to use some feasible algorithm - e.g., solving a system of linear equations - to replace the original plan $\widetilde{x}$ with a modified plan $\widetilde{x}+\Delta x$. 
Due to NP-hardness, this feasibly modified plan is, in general, not optimal, but we hope that the resulting loss $L_{1} \stackrel{\text { def }}{=} u\left(x^{\mathrm{opt}}, \widetilde{a}+\Delta a\right)-u(\widetilde{x}+\Delta x, \widetilde{a}+\Delta a)$ is much smaller than the loss $L_{0}$ corresponding to the use of the original plan $\widetilde{x}$.

What we do in this paper. In this paper, we analyze the values of both losses and we show that indeed, $L_{1}$ is much smaller than $L_{0}$. So, in many situations, even if the loss $L_{0}$ is so large than the corresponding strategy (of directly using the original plan) is worthless, the modified plan may leads to a reasonably small loss $L_{1} \ll L_{0}$ - thus explaining Eisenhower's observation.

Estimating $L_{0}$. We assume that the difference $\Delta a$ is reasonably small, so the corresponding difference in action $\Delta x^{\text {opt }} \stackrel{\text { def }}{=} x^{\text {opt }}-\widetilde{x}$ is also small. We can therefore expand the expression for the loss $L_{0}$ in Taylor series and keep only terms which are linear and quadratic with respect to $\Delta x$. Thus, we get

$$
\begin{gathered}
L_{0}=u\left(x^{\mathrm{opt}}, \widetilde{a}+\Delta a\right)-u\left(x^{\mathrm{opt}}-\Delta x^{\mathrm{opt}}, \widetilde{a}+\Delta a\right)= \\
\sum_{i=1}^{n} \frac{\partial u}{\partial x_{i}}\left(x^{\mathrm{opt}}, \widetilde{a}+\Delta a\right) \cdot \Delta x_{i}^{\mathrm{opt}}+ \\
\frac{1}{2} \cdot \sum_{i=1}^{n} \sum_{i^{\prime}=1}^{n} \frac{\partial^{2} u}{\partial x_{i} \partial x_{i^{\prime}}}\left(x^{\mathrm{opt}}, \widetilde{a}+\Delta a\right) \cdot \Delta x_{i}^{\mathrm{opt}} \cdot \Delta x_{i^{\prime}}^{\mathrm{opt}}+o\left((\Delta a)^{2}\right) .
\end{gathered}
$$

By definition, the action $x^{\text {opt }}$ maximizes the utility $u(x, \widetilde{a}+\Delta a)$. Thus, we have $\frac{\partial u}{\partial x_{i}}\left(x^{\mathrm{opt}}, \widetilde{a}+\Delta a\right)=0$, and the above expression for the loss $L_{0}$ takes the simplified form

$$
L_{0}=\frac{1}{2} \cdot \sum_{i=1}^{n} \sum_{i^{\prime}=1}^{n} \frac{\partial^{2} u}{\partial x_{i} \partial x_{i^{\prime}}}\left(x^{\mathrm{opt}}, \widetilde{a}+\Delta a\right) \cdot \Delta x_{i}^{\mathrm{opt}} \cdot \Delta x_{i^{\prime}}^{\mathrm{opt}}+o\left((\Delta a)^{2}\right) .
$$

The values $\Delta x_{i}^{\text {opt }}$ can be estimated from the above condition

$$
\frac{\partial u}{\partial x_{i}}\left(x^{\mathrm{opt}}, \widetilde{a}+\Delta a\right)=\frac{\partial u}{\partial x_{i}}\left(\widetilde{x}+\Delta x^{\mathrm{opt}}, \widetilde{a}+\Delta\right)=0 .
$$

Expanding this expression in Taylor series in terms of $\Delta x_{i}$ and $\Delta a_{j}$ and taking into account that $\frac{\partial u}{\partial x_{i}}(\widetilde{x}, \widetilde{a})=0$ (since for $a=\widetilde{a}$, the utility is maximized by the action $x=\widetilde{x}$ ), we conclude that for every $i$, we have

$$
\sum_{i^{\prime}=1}^{n} \frac{\partial^{2} u}{\partial x_{i} \partial x_{i^{\prime}}}(\widetilde{x}, \widetilde{a}) \cdot \Delta x_{i^{\prime}}^{\mathrm{opt}}+\sum_{j=1}^{m} \frac{\partial^{2} u}{\partial x_{i} \partial a_{j}}(\widetilde{x}, \widetilde{a}) \cdot \Delta a_{j}+o(\Delta x, \Delta a)=0 .
$$

Thus, the first approximation $\Delta x_{i}$ to the values $\Delta x_{i}^{\text {opt }}$ can be determined as a solution to a system of linear equations:

$$
\sum_{i^{\prime}=1}^{n} \frac{\partial^{2} u}{\partial x_{i} \partial x_{i^{\prime}}}(\widetilde{x}, \widetilde{a}) \cdot \Delta x_{j}=-\sum_{j=1}^{m} \frac{\partial^{2} u}{\partial x_{i} \partial a_{j}}(\widetilde{x}, \widetilde{a}) \cdot \Delta a_{j} .
$$


A solution to a system of linear equations is a linear combination of the righthand sides. Thus, the values $\Delta x_{i}$ are a linear function of $\Delta a_{j}$. Substituting these linear expressions into the formula (1), we conclude that the loss $L_{0}$ is a quadratic function of $\Delta a_{j}$, i.e., that $L_{0}=\sum_{j=1}^{m} \sum_{j^{\prime}=1}^{m} k_{j j^{\prime}} \cdot \Delta a_{j} \cdot \Delta a_{j^{\prime}}+o\left((\Delta a)^{2}\right)$ for some coefficients $k_{j j^{\prime}}$.

Estimating $L_{1}$. In the previous section, we considered what happens if we use the original plan $\widetilde{x}$ - which was optimal in the original situation $\widetilde{a}-$ in the changed situation $a=\widetilde{a}+\Delta a$. Since the original plan is optimal only for the original situation, but not for the new one, using this not-optimal plan leads to the loss $L_{0}$, a loss which we estimated as being quadratic in terms of $\Delta a$.

To decrease this loss, we need to update the action $x$. As we have already mentioned, exactly computing the optimal action $x^{\text {opt }}$ is, in general, an NPhard - i.e., computationally intractable - problem. However, as we have also mentioned, the first approximation $\Delta x_{i}$ to the desired difference $\Delta x^{\mathrm{opt}}-$ and thus, the first approximation to the newly optimal solution $x^{\text {opt }}$ - can be obtained by solving a system of linear equations (2).

The system (2) of linear equations is feasible to solve. Thus, it is reasonable to consider using the action $x^{\text {lin }}=\widetilde{x}+\Delta x$ instead of the original action $\widetilde{x}$. Let us estimate how much we lose if we use this new action $x^{\text {lin }}$ instead of the optimal action $x_{i}^{\text {opt }}$.

The fact that the difference $\Delta x$ is the first approximation to the optimal difference $\Delta x^{\mathrm{opt}}$ means that we can write $\Delta x^{\mathrm{opt}}=\Delta x+\delta x$, where the remaining term $\delta x \stackrel{\text { def }}{=} \Delta x^{\mathrm{opt}}-\Delta x=x^{\mathrm{opt}}-x^{\mathrm{lin}}$ is of second order in terms of $\Delta x$ and $\Delta a$ : $\delta x=O\left((\Delta x)^{2},(\Delta a)^{2}\right)$. Since in the first approximation, $\Delta x$ has the same order as $\Delta a$, we thus get $\delta x=O\left((\Delta a)^{2}\right)$.

The loss $L_{1}$ of using $x^{\text {lin }}=x^{\text {opt }}-\delta x$ instead of $x^{\text {opt }}$ is equal to the difference $L_{1}=u\left(x^{\mathrm{opt}}, \widetilde{a}+\Delta a\right)-u\left(x^{\mathrm{lin}}, \widetilde{a}+\Delta a\right)=u\left(x^{\mathrm{opt}}, \widetilde{a}+\Delta a\right)-u\left(x^{\mathrm{opt}}-\delta x, \widetilde{a}+\Delta a\right)$.

If we expand this expression in $\delta x$ and keep only linear and quadratic terms, we conclude that

$$
\begin{gathered}
L_{1}=\sum_{i=1}^{n} \frac{\partial u}{\partial x_{i}}\left(x^{\mathrm{opt}}, \widetilde{a}+\Delta a\right) \cdot \delta x_{i}+ \\
\frac{1}{2} \cdot \sum_{i=1}^{n} \sum_{i^{\prime}=1}^{n} \frac{\partial^{2} u}{\partial x_{i} \partial x_{i^{\prime}}}\left(x^{\mathrm{opt}}, \widetilde{a}+\Delta a\right) \cdot \delta x_{i} \cdot \delta x_{i^{\prime}}+o\left((\delta x)^{2}\right) .
\end{gathered}
$$

Since $x^{\text {opt }}$ is the action that, for $a=\widetilde{a}+\Delta a$, maximizes utility, we get

$$
\frac{\partial u}{\partial x_{i}}\left(x^{\mathrm{opt}}, \widetilde{a}+\Delta a\right)=0 .
$$

Thus, the expression for $L_{1}$ gets a simplified form

$$
L_{1}=\frac{1}{2} \cdot \sum_{i=1}^{n} \sum_{i^{\prime}=1}^{n} \frac{\partial^{2} u}{\partial x_{i} \partial x_{i^{\prime}}}\left(x^{\mathrm{opt}}, \widetilde{a}+\Delta a\right) \cdot \delta x_{i} \cdot \delta x_{i^{\prime}}+o\left((\delta x)^{2}\right) .
$$


We know that the values $\delta x_{i}$ are quadratic in $\Delta a$; thus, we conclude that for the modified action, the loss $L_{1}$ is a 4 -th order function of $\Delta a_{j}$, i.e., that

$$
L_{1}=\sum_{j=1}^{m} \sum_{j^{\prime}=1}^{m} \sum_{j^{\prime \prime}=1}^{m} \sum_{j^{\prime \prime \prime}=1}^{m} k_{j j^{\prime} j^{\prime \prime} j^{\prime \prime \prime}} \cdot \Delta a_{j} \cdot \Delta a_{j^{\prime}} \cdot \Delta a_{j^{\prime \prime}} \cdot \Delta a_{j^{\prime \prime \prime}}+o\left((\Delta a)^{5}\right)
$$

for some coefficients $k_{j j^{\prime} j^{\prime \prime} j^{\prime \prime \prime}}$.

\section{Conclusions}

We conclude that:

- the loss $L_{0}$ related to using the original plan is quadratic in $\Delta a$, while

- the loss $L_{1}$ related to using a feasibly modified plan is of 4 th order in terms of $\Delta a$.

For reasonably small $\Delta a$, we have $L_{1} \sim(\Delta a)^{4} \ll L_{0} \sim(\Delta a)^{2}$.

Let $\varepsilon>0$ be the maximum loss that we tolerate. Since $L_{1} \ll L_{0}$, we have three possible cases: (1) $\varepsilon<L_{1}$, (2) $L_{1} \leq \varepsilon \leq L_{0}$, and (3) $L_{0}<\varepsilon$. In the first case, even using the modified action does not help. In the third case, the change in the situation is so small that it is Ok to use the original plan $\widetilde{x}$.

In the second case, we have exactly the Eisenhower situation:

- if we use the original plan $\widetilde{x}$, the resulting loss $L_{0}$ much larger than we can tolerate; in this sense, the original plan is worthless;

- on the other hand, if we feasible modify the original plan into $x^{\text {lin }}$, then we get an acceptable action.

So, we indeed get a theoretical justification of Eisenhower's observation.

Acknowledgments. This work was supported in part by the National Science Foundation grants HRD-0734825 and HRD-1242122 (Cyber-ShARE Center of Excellence) and DUE-0926721, and by an award "UTEP and Prudential Actuarial Science Academy and Pipeline Initiative" from Prudential Foundation.

\section{References}

1. D. Eisenhower, "A speech to the National Defense Executive Reserve Conference in Washington, D.C., November 14, 1957", in: D. Eisenhower, Public Papers of the Presidents of the United States, National Archives and Records Service, Government Printing Office, 1957, p. 818.

2. V. Kreinovich, A. Lakeyev, J. Rohn, and P. Kahl, Computational Complexity and Feasibility of Data Processing and Interval Computations, Kluwer, Dordrecht, 1998.

3. R. D. Luce and R. Raiffa, Games and Decisions: Introduction and Critical Survey, Dover, New York, 1989.

4. H. T. Nguyen, O. Kosheleva, and V. Kreinovich, "Decision making beyond Arrow's 'impossibility theorem', with the analysis of effects of collusion and mutual attraction", International Journal of Intelligent Systems, 2009, Vol. 24, No. 1, pp. 27-47.

5. P. Pardalos, Complexity in Numerical Optimization, World Scientific, Singapore, 1993. 